\title{
GREEN'S FUNCTION FOR A PIECEWISE CONTINUOUS POTENTIAL VIA INTEGRAL EQUATIONS METHOD
}

\author{
Brahim Benali ${ }^{1},{\text { Mohammed Tayeb } \text { Meftah }^{2}, \text { Rai Vandana }}^{3}$ \\ ${ }^{1}$ Department of Mathematics, Faculty of Exact Sciences and LABTHOP \\ Laboratory, University Hamma Lakhadar, EL-oued 39000 Algeria, \\ E-mail: benalibrahim@ymail.com \\ ${ }^{2}$ Department of Matter Sciences, Faculty of Mathematics and Matter \\ Sciences and LRPPS Laboratory, University of Kasdi Merbah, Ouargla \\ 30000 Algeria, \\ E-mail: mewalid@yahoo.com \\ ${ }^{3}$ Department of Management Studies, Indian Institute of Technology, \\ Madras, Chennai 600 036, Tamil Nadu, India, \\ E-mail: vdrai1988@gmail.com
}

\begin{abstract}
The aim of this work is to provide Green's function for the Schrodinger equation. The potential part in the Hamiltonian is piecewise continuous operator. It is a zero operator on a disk of radius "a" and a constant $V_{0}$ outside this disk (in two dimensions). We have used, to construct the Green's function, the technique of the integral equations. We have respected the boundary conditions of the problem. The discrete spectra of the Hamiltonian operator have been also derived.

integral equations and Green Kernel and Bessel Transformation:
\end{abstract}

\begin{abstract}
Abstrak. Tujuan utama dari penelitian ini adalah menyediakan fungsi Green untuk persamaan Schrodinger. Bagian energi potensial dalam rumusan Hamiltoniannya adalah operator yang piecewise continue. Operator tersebut merupakan suatu operator nol pada cakram berjari-jari "a" dan berjari-jari suatu konstanta $V_{0}$ di luar cakram terkait (dalam dimensi dua). Di dalam konsruksi fungsi Green, digunakan teknik persamaan integral. Kondisi batas dari masalah juga diperhatikan. Spektra diskrit dari operator Hamiltonian juga diberikan.
\end{abstract}

Kata kunci: Persamaan integral, Kernel Green, Transformasi Bessel

2000 Mathematics Subject Classification: 03.75.Lm and 03.65.Db and 02.30.Gp and 02.30.Sa Received: 22-12-2016, revised: 02-02-2018 accepted: 20-02-2018. 


\section{INTRODUCTION}

Green's functions are very usefull in the studies of linear partial differential equations and are widely studied from the point of view of fundamental solutions of these equations. They provide a general method for solving the linear differential equations or equivalentely the integral equations. They are widely used in quantum mechanics to discover the energy spectra of physical system. In mathematics the term Green's functions is often given to solutions of an initial- or boundary-value problem of a linear differential equation with a $\delta$-function as an inhomogeneous term. Some times we encounter, in various fields of mathematics, physics, applied physics, and engineering, the terms: propagator, resolvent, resolvent kernel, signal function, point response function, or transfer function. These terms are nothing but the Green functions spelled differentely with respect of the field. Strictely saying, the Green function serves to find the output for a given input. For this reason, one can understand why Greens functions are very useful in many fields: in electromagnetism, hydrodynamics, acoustics, elasticity, quantum mechanics, elementary-particle physics, etc.... Their usefulness is still growing ascendentely today, as various numerical techniques increase to develop the calculations of Greens functions. Recall that there are usually several Greens functions associated with the same equation. These difference is related to the boundary conditions. Therefore it is important, when we search the Green function of the linear differential equation, to specify the boundary conditions.

Before giving the directive of our paper, it is necessary to mention some works which are closely related to our investigation. In [1], the Green's function has been studied for the thin circular Kirchhoff Poisson-plate. The Green's function has been also studied by [2] in three dimensions for a piecewise continuous potential possessing a spherical symmetry. In [3]-[4] the Green's function has been analyzed for the elliptic domain. The quantum problem relative to scattering in two dimensions was also treated asymptotically in [5]. Using the approximative methods, [7]- [6][8]- [9] have treated the Green's function problem. In this work we shall present, an explicit calculation of the Greens function, for a piecewise continuous potential presenting a circular symmetry. The technique of the integral equations and their solutions with the help of the Bessel's transform are the main results of this work. In our work, we address the problem of a time-independant Schroedinger equation on a disk with a piecewise continuous potential presenting a circular symmetry. In quantum mechanics, if the potential is constant in a disk and equal to zero outside (or vice versa) the solution of the time-independant Schroedinger equation is continuous and its derivative is discontinuous on the boundary (the edge) of the disk. Specify one more thing in our problem: the time-independant Schroedinger equation takes two different forms depending on whether it is inside the disk or outside. This kind of problem matches in quantum mechanics to the study of a particle subject to a potential which is a piecewise continuous possessing a circular symmetry.

In Section 2, we present a theoretical framework in which our work is focuced. In 
particular we show how the Schroedinger equation transforms to an integral equation and how the latter can be solved using the Bessel's transform. In section 3, we calculate Greens functions corresponding to the potential zero on a disk and equal to positive constant outside of the disk. It arises that the Bessel's transform is adequate to solve this kind of problems. In section 4, using the Bessel's transform, we calculate Greens functions corresponding to the potential equal to a negative constant in the disk equal to zero outside of the disk. At the end, we finish this work by conclusions in Section 5 .

\section{THEORETICAL PRELIMINARIES}

2.1. Schroedinger equation. At first we consider the almost fundamental equation in physics: the Schroedinger equation in one dimension $(D=1)$ :

$$
(-\Delta+2 m V(x)) \Psi(x, t)=2 m i \frac{\partial \Psi}{\partial t}
$$

This equation is annouced at the earlier of the development of modern physics about one century ago. $\Delta$ is the Laplacian in one dimension. $V(x)$ must be a measurable, real-valued and locally bounded function to ensure the regularity of $\psi(x, t)[10]$. Its solution can be formally written as [11]:

$$
\Psi(x, t)=\int_{-\infty}^{+\infty} K(x, t: y, 0) \Psi(y, 0) d y
$$

where $\Psi(y, 0)$ is the solution of (1) at $t=0$ that is to say the initial condition of the problem of Schroedinger equation. $K(x, t: y, 0)$ is the kernel of the problem (2.1)(some theoretical physists name it the propagator or sometimes Green's function, bien que la fonction de Green est la transforme de Fourrier de K par rapport $\mathrm{t})$. We need in the following, the free kernel $K_{0}(x, t: y, 0)$ coresponding to the free Shroedinger equation $-\Delta \Psi(x, t)=2 m i \partial \Psi / \partial t$. The research of the solution of the Schrodinger equation (1), turn out then to calculate the Green's function satisfying the following integral equation:

$$
g(x, E: y)=g_{0}(x, E: y)-\int_{-\infty}^{+\infty} d \zeta\left[g_{0}(x, E: \zeta) V(\zeta) g(\zeta, E: y)\right]
$$

where $g(x, E: y)$ and $g_{0}(x, E: y)$ are Fourrier transforms of $K(x, t: y, 0)$ and $K_{0}(x, t: y, 0)$ respectively:

$$
\begin{aligned}
g(x, E: y) & =\int_{-\infty}^{+\infty} K(x, t: y, 0) \exp (i E t) d t \\
g_{0}(x, E: y) & =\int_{-\infty}^{+\infty} K_{0}(x, t: y, 0) \exp (i E t) d t
\end{aligned}
$$

To solve the integral equation (3), we shall give, in the next step, some theoretical tools. 
2.2. Hilbert's problem. Let $L$ be an infinite line whose equation is $\operatorname{Re}(s)=b$. Hilbert's problem consists to find a piecewise analytic function $\varphi$ piecewise analytic with respect to the line $L$. We demand in further that this function has an incresing behavior not great than polynomial at infinity and verifies the following equation:

$$
\Phi_{i}(z)=A(z) \Phi_{e}(z)+B(z), \quad z \epsilon L,
$$

where $A(z)$ and $B(z)$ are functions defined on the line $L$. Let $\Phi_{i}(s)$ and $\Phi_{e}(s)$ are left and wright limits of $\Phi(s)$ solution of homgeneous Hilbert problem $(B \equiv 0)$. We assume, in addition, that $A(z)$ have not zeros on the line $L$.

\section{Hilbert's Theorem}

Consider $\mathrm{A}(\mathrm{z})$ a Holderian function on the line $L$ whose equation is $\operatorname{Re}(z)=b$, such that $\mathrm{A}(\mathrm{z})$ not vanishes and verifies

$$
|A(z)-1| \leq \frac{C}{|z|^{\gamma}}, \quad \gamma>0, \quad z \epsilon L,
$$

and consider $\mathrm{B}(\mathrm{z})$ a second Holderian function on $L$ such that

$$
|B(z)-B(\infty)| \leq \frac{C}{|z|^{\gamma}}, \quad \gamma>0, \quad z \epsilon L .
$$

then the only piecewise analytic solutions on the line $L$ of Hilbert's problem (4) are given by:

$$
\varphi(z)=\frac{\Phi_{i}(z)}{2 \pi i} \oint_{b-i \infty}^{b+i \infty} \frac{B(s)}{\Phi_{i}(s)(s-z)} d s+\mathbf{P}(z) \Phi(z), \quad z \notin L
$$

where $\Phi$ is the solution of the homogeneous Hilbert problem and $\Phi_{i}$ is given (for any $z \in L$ ) by

$$
\begin{array}{r}
\log \left[(z-\beta)^{p} \Phi_{i}(z)\right]=\frac{1}{2} \log \left(A(z)\left(\frac{z-\beta}{z-\alpha}\right)^{p}\right) \\
+\frac{1}{2 \pi i} \oint_{b-i \infty}^{b+i \infty} \log \left(A(s)\left(\frac{s-\beta}{s-\alpha}\right)^{p}\right) \frac{d s}{s-z}
\end{array}
$$

$\mathbf{P}(\mathbf{z})$ is some polynomial, and $\alpha, \beta$ are complexe numbers such that $\operatorname{Re}(\alpha)>b$ and $\operatorname{Re}(\beta)<b[12]$

\section{TWO-DIMENSIONS PROBLEMS}

Consider the problem relative to the Schrödinger equation where the potentiel term is a piecewise continuous: constant (zero) on a disk of radius "a" and equal to a constant $V_{0}$ outside the disk. Let the following integral equation

$$
g_{l}\left(r, r^{\prime}\right)=g_{0}\left(r, r^{\prime}\right)-V_{0} \int_{a}^{\infty} \zeta g_{0}(r, \zeta) g_{l}\left(\zeta, r^{\prime}\right) d \zeta
$$


In view to solve the last integral equation, define new pair functions $g_{l}^{+}(p, r)$ and $g_{l}^{-}(p, r)$ such that

$$
\begin{gathered}
g_{l}^{+}(p, r)=\left\{\begin{array}{cc}
g_{l}(p, r) & \quad r<a \\
0 & r \geq a
\end{array}\right. \\
g_{l}^{-}(p, r)=\left\{\begin{array}{cl}
0 & r<a \\
-g_{l}(p, r) & r \geq a
\end{array}\right.
\end{gathered}
$$

then the integral equation (9) transforms as

$$
g_{l}^{+}\left(r, r^{\prime}\right)-g_{l}^{-}\left(r, r^{\prime}\right)=g_{0}\left(r, r^{\prime}\right)+V_{0} \int_{0}^{\infty} \zeta g_{0}(r, \zeta) g_{l}^{-}\left(\zeta, r^{\prime}\right) d \zeta
$$

Define Hankel's transform of a function $g_{l}\left(r, r^{\prime}\right)$ viewed as a function of the variable "r" by the following expression

$$
\hat{G}_{l}\left(p, r^{\prime}\right)=\int_{0}^{\infty} r J_{l}(p r) g_{l}\left(r, r^{\prime}\right) d r
$$

The Hankel transform applied to the equation (12) gives

$$
\begin{array}{r}
\int_{0}^{\infty} r J_{l}(p r) g_{l}^{+}\left(r, r^{\prime}\right) d r-\int_{0}^{\infty} r J_{l}(p r) g_{l}^{-}\left(r, r^{\prime}\right) d r= \\
\left.\int_{0}^{\infty} r J_{l} p r\right) g_{0}\left(r, r^{\prime}\right) d r+V_{0} \int_{0}^{\infty} r J_{l}(p r) d r \int_{a}^{\infty} \zeta g_{0}(r, \zeta) g_{l}^{-}\left(\zeta, r^{\prime}\right) d \zeta
\end{array}
$$

A integral representation of the kernel $g_{0}\left(r, r^{\prime}\right)$ is given by [13]

$$
g_{0}\left(r, r^{\prime}\right)=\int_{0}^{\infty} \exp \left[-2 \alpha \epsilon\left(\frac{r^{2}+r^{\prime 2}}{u}\right)+\frac{u}{2}\right] I_{l}\left(4 \alpha \epsilon \frac{r r^{\prime}}{u}\right) \frac{d u}{u}
$$

by substituting (15) in (12) we obtain

$$
\begin{array}{r}
\hat{G}_{l}^{+}\left(p, r^{\prime}\right)-\hat{G}_{l}^{-}\left(p, r^{\prime}\right)=\int_{0}^{\infty} \exp \left[-2 \alpha \epsilon\left(\frac{r^{\prime 2}}{u}\right)+\frac{u}{2}\right] \frac{d u}{u} \\
\int_{0}^{\infty} r J_{l}(p r) I_{l}\left(4 \alpha \epsilon \frac{r r^{\prime}}{u}\right) \exp \left(\frac{-2 \alpha \epsilon r^{2}}{u}\right) d r \\
+V_{0} \int_{0}^{\infty} \int_{0}^{\infty} \exp \left[-2 \alpha \epsilon\left(\frac{\zeta^{2}}{u}\right)+\frac{u}{2}\right] \frac{d u}{u} \zeta g_{l}^{-}\left(\zeta, r^{\prime}\right) d \zeta \\
\int_{0}^{\infty} r J_{l}(p r) I_{l}\left(4 \alpha \epsilon \frac{r \zeta}{u}\right) \exp \left(\frac{-2 \alpha \epsilon r^{2}}{u}\right) d r
\end{array}
$$


Using formula 6.633.4 [14], it is straighforward that

$$
\begin{gathered}
\hat{G}_{l}^{+}\left(p, r^{\prime}\right)-\hat{G}_{l}^{-}\left(p, r^{\prime}\right)= \\
\int_{0}^{\infty} \exp \left[-2 \alpha \epsilon\left(\frac{16 \alpha^{2} \epsilon^{2}}{8 \alpha \epsilon}-2 \alpha \epsilon\right) \frac{r^{\prime 2}}{u}+\frac{u}{2}\left(1-\frac{p^{2}}{4 \alpha \epsilon}\right)\right] J_{l}\left(p r^{\prime}\right) \frac{d u}{4 \alpha \epsilon} \\
+V_{0} \int_{0}^{\infty} \zeta d \zeta \int_{0}^{\infty} \exp \left[-2 \alpha \epsilon\left(\frac{16 \alpha^{2} \epsilon^{2}}{8 \alpha \epsilon}-2 \alpha \epsilon\right) \frac{\zeta^{2}}{u}+\frac{u}{2}\left(1-\frac{p^{2}}{4 \alpha \epsilon}\right)\right] \\
J_{l}(p \zeta) g_{l}^{-}\left(\zeta, r^{\prime}\right) \frac{d u}{4 \alpha \epsilon}
\end{gathered}
$$

or equivalently

$$
\begin{array}{r}
\hat{G}_{l}^{+}\left(p, r^{\prime}\right)-\hat{G}_{l}^{-}\left(p, r^{\prime}\right)= \\
=\int_{0}^{\infty} \exp \left[\left(1-\frac{p^{2}}{4 \alpha \epsilon}\right) \frac{u}{2}\right] \frac{d u}{4 \alpha \epsilon}\left[J_{l}\left(p r^{\prime}\right)+V_{0} \int_{0}^{\infty} \zeta J_{l}(p \zeta) g_{l}^{-}\left(\zeta, r^{\prime}\right) d \zeta\right]
\end{array}
$$

Under the condition $1-\frac{p^{2}}{4 \alpha \epsilon}<0$, the first integral in the last equation converges and gives

$$
\hat{G}_{l}^{+}\left(p, r^{\prime}\right)-\hat{G}_{l}^{-}\left(p, r^{\prime}\right)=\frac{2}{\left(p^{2}-4 \alpha \epsilon\right)}\left[J_{l}\left(p r^{\prime}\right)+V_{0} \int_{0}^{\infty} \zeta J_{l}(p \zeta) g_{l}^{-}\left(\zeta, r^{\prime}\right) d \zeta\right]
$$

then the integral equation (12) transforms under Hankel's transform as

or

$$
\hat{G}_{l}^{+}\left(p, r^{\prime}\right)-\hat{G}_{l}^{-}\left(p, r^{\prime}\right)=\frac{2 J_{l}\left(p r^{\prime}\right)}{p^{2}-4 \alpha \epsilon}+\frac{2 V_{0}}{p^{2}-4 \alpha \epsilon} \hat{G}_{l}^{-}\left(p, r^{\prime}\right)
$$

By using the abbreviations

$$
\hat{G}_{l}^{+}\left(p, r^{\prime}\right)-\left[1+\frac{2 V_{0}}{p^{2}-4 \alpha \epsilon}\right] \hat{G}_{l}^{-}\left(p, r^{\prime}\right)=\frac{2 J_{l}\left(p r^{\prime}\right)}{p^{2}-4 \alpha \epsilon}
$$

$$
\epsilon=E, \quad 2 \alpha=m, \quad \text { we have } \quad 4 \alpha \epsilon=2 m E=k^{2}, \quad \mu^{2}=k^{2}-2 V_{0}
$$

the equation (21) writes as

$$
\hat{G}_{l}^{+}\left(p, r^{\prime}\right)-\left[\frac{p^{2}-\mu^{2}}{p^{2}-k^{2}}\right] \hat{G}_{l}^{-}\left(p, r^{\prime}\right)=\frac{2 J_{l}\left(p r^{\prime}\right)}{p^{2}-k^{2}}=b(p)
$$

Now, consider the decomposition [15]

and put

$$
\frac{p^{2}-\mu^{2}}{p^{2}-k^{2}}=\frac{(p+\mu)(p-\mu)}{(p+k)(p-k)}
$$




$$
\chi^{+}(p)=\frac{p-\mu}{p-k}, \quad \text { and } \quad \chi^{-}(p)=\frac{p+k}{p+\mu}
$$

The Hilbert's theorem asserts that the functions $\hat{G}_{l}^{+}$and $\hat{G}_{l}^{-}$are determinated by the case $r^{\prime}>a$ :

$$
\begin{aligned}
\hat{G}_{l}^{+}\left(p, r^{\prime}\right)=\frac{\chi^{+}(p)}{2 \pi i} & \int_{L} \frac{b(\tau) d \tau}{\chi^{+}(\tau)(\tau-p)}, \quad \text { where } L \text { is a closed contour } \\
& =\frac{2(p-\mu)}{2 \pi i(p-k)} \int_{L} \frac{J_{l}\left(\tau r^{\prime}\right) d \tau}{\left(\tau^{2}-k^{2}\right)\left(\frac{\tau-\mu}{\tau-k}\right)(\tau-p)} \\
& =\frac{2(p-\mu)}{2 \pi i(p-k)} \int_{L} \frac{J_{l}\left(\tau r^{\prime}\right) d \tau}{(\tau+k)(\tau-\mu)(\tau-p)}
\end{aligned}
$$

By using the residue theorem and apply it at the points $\tau=-k$ and $\tau=p$ inside the contour, we obtain

$$
\begin{aligned}
\hat{G}_{l}^{+}\left(p, r^{\prime}\right) & =\frac{2 B(k, \mu, a)(p-\mu) J_{l}\left(k r^{\prime}\right)}{(p-k)(\mu+k)(p+k)}+\frac{2(p-\mu) J_{l}\left(p r^{\prime}\right)}{(p-k)(p+k)(p-\mu)} \\
& =\frac{2 J_{l}\left(p r^{\prime}\right)}{(p-k)(p+k)}+\frac{2 B(k, \mu, a)(p-\mu) J_{l}\left(k r^{\prime}\right)}{(p-k)(\mu+k)(p+k)}
\end{aligned}
$$

The inverse Hankel's transform applied to the last equation for $r^{\prime}>r>a$ gives

$$
\begin{aligned}
g_{l}^{+,+}\left(r, r^{\prime}\right)= & 2 \int_{0}^{+\infty} \frac{p J_{l}(p r) J_{l}\left(p r^{\prime}\right) d p}{(p-k)(p+k)}+ \\
& \frac{2 B(k, \mu, a) J_{l}\left(k r^{\prime}\right)}{(\mu+k)} \int_{0}^{+\infty} \frac{p(p-\mu) J_{l}(p r) d p}{\left(p^{2}-k^{2}\right)} \\
= & -\pi J_{l}\left(k r^{\prime}\right) Y_{l}(k r)+\frac{2 B(k, \mu, a) J_{l}\left(k r^{\prime}\right) J_{l}(k r)}{(\mu+k)}
\end{aligned}
$$

the same technique used for the case $r^{\prime}<a$ gives:

$$
\begin{gathered}
\hat{G}_{l}^{-}\left(p, r^{\prime}\right)=\frac{\chi^{-}(p)}{2 \pi i} \int_{L} \frac{b(\tau) d \tau}{\chi^{+}(\tau)(\tau-p)}, \quad \text { where } L \text { is a closed contour } \\
=\frac{2(p+k)}{2 \pi i(p+\mu)} \int_{L} \frac{J_{l}\left(\tau r^{\prime}\right) d \tau}{(\tau+k)(\tau-\mu)(\tau-p)}
\end{gathered}
$$

Respecting the residue theorem for the points $\tau=\mu$ and $\tau=p$ inside the contour, we find 


$$
\begin{aligned}
\hat{G}_{l}^{-}\left(p, r^{\prime}\right) & =\frac{2(p+k) J_{l}\left(p r^{\prime}\right)}{(p+\mu)(p+k)(p-\mu)}+\frac{2 A(k, \mu, a)(p+k) J_{l}\left(\mu r^{\prime}\right)}{(p+\mu)(\mu+k)(p-\mu)} \\
& =\frac{2 A(k, \mu, a)(p+k) J_{l}\left(\mu r^{\prime}\right)}{(p+\mu)(\mu+k)(p-\mu)}+\frac{2 J_{l}\left(p r^{\prime}\right)}{(p+\mu)(p-\mu)}
\end{aligned}
$$

The inverse Hankel's transform applied to the last equation for $r<r^{\prime}<a$ gives:

$$
\begin{aligned}
g_{l}^{-,-}\left(r, r^{\prime}\right)= & \frac{2 A(k, \mu, a) J_{l}\left(\mu r^{\prime}\right)}{(\mu+k)} \int_{0}^{+\infty} \frac{p(p+k) J_{l}(p r) d p}{p^{2}-\mu^{2}}+ \\
& 2 \int_{0}^{+\infty} \frac{p J_{l}(p r) J_{l}\left(p r^{\prime}\right) d p}{p^{2}-\mu^{2}} \\
= & \frac{2 A(k, \mu, a) J_{l}\left(\mu r^{\prime}\right) J_{l}(\mu r)}{(\mu+k)}-\pi J_{l}\left(\mu r^{\prime}\right) Y_{l}(\mu r)
\end{aligned}
$$

the continuity of Green's function at $r=a$ allows to write:

$$
g_{l}^{+,+}\left(a, r^{\prime}\right)=g_{l}^{-,-}\left(a, r^{\prime}\right)
$$

implies

$$
\begin{aligned}
- & \pi J_{l}\left(k r^{\prime}\right) Y_{l}(k r)+\frac{2 B(k, \mu, a) J_{l}\left(k r^{\prime}\right) J_{l}(k r)}{(\mu+k)}= \\
& \frac{2 A(k, \mu, a) J_{l}\left(\mu r^{\prime}\right) J_{l}(\mu r)}{(\mu+k)}-\pi J_{l}\left(\mu r^{\prime}\right) Y_{l}(\mu a)
\end{aligned}
$$

the derivability gives

$$
\begin{gathered}
\left(\frac{\partial g_{l}^{+,+}\left(a, r^{\prime}\right)}{\partial r}\right)_{r=a}=\left(\frac{\partial g_{l}^{-,-}\left(a, r^{\prime}\right)}{\partial r}\right)_{r=a} \\
k\left(-\pi J_{l}\left(k r^{\prime}\right) Y_{l}^{\prime}(k r)+\frac{2 B(k, \mu, a) J_{l}\left(k r^{\prime}\right) J_{l}^{\prime}(k r)}{(\mu+k)}\right) \\
=\mu\left(\frac{2 A(k, \mu, a) J_{l}\left(\mu r^{\prime}\right) J_{l}^{\prime}(\mu r)}{(\mu+k)}-\pi J_{l}\left(\mu r^{\prime}\right) Y_{l}^{\prime}(\mu a)\right)
\end{gathered}
$$

Using the symmetry properties of the Green's function, we take $A, B$ two functions depending on $a$, we obtain the following system at $r=r^{\prime}=a$

$$
\begin{array}{r}
g_{l}^{++}(a, a)=g_{l}^{--}(a, a) \\
\left(\frac{d g_{l}^{++}(r, a)}{d r}\right)_{r=a}=\left(\frac{d g_{l}^{--}(r, a)}{d r}\right)_{r=a}
\end{array}
$$

that transformed as a system of two coupled equations, the first one is

$$
\begin{array}{r}
\quad \pi(\mu+k) J_{l}(k a) Y_{l}(k a)+2 B(k, \mu, a) J_{l}(k a) J_{l}(k a) \\
=2 A(k, \mu, a) J_{l}(\mu a) J_{l}(\mu a)-\pi(\mu+k) J_{l}(\mu a) Y_{l}(\mu a)
\end{array}
$$


and the second is

$$
\begin{array}{r}
\quad \pi k(\mu+k) Y_{l}^{\prime}(k a) J_{l}(k a)+2 k B(k, \mu, a) J_{l}^{\prime}(k a) J_{l}(k a) \\
=2 \mu A(k, \mu, a) J_{l}(\mu a) J_{l}^{\prime}(\mu a)-\mu \pi(\mu+k) Y_{l}^{\prime}(\mu a) J_{l}(\mu a)
\end{array}
$$

performing some calculus we find

$$
A(k, \mu, a)=(\mu+k) Z_{1}(k, \mu, a)
$$

where

$$
Z_{1}(k, \mu, a)=\frac{-2 J_{l}(k a)+\pi a J_{l}(\mu a)\left(k J_{l}^{\prime}(k a) Y_{l}(\mu a)-\mu J_{l}(k a) Y_{l}^{\prime}(\mu a)\right)}{2 a J_{l}(\mu a)\left[k J_{l}^{\prime}(k a) J_{l}(\mu a)-\mu J_{l}(k a) J_{l}^{\prime}(\mu a)\right]}
$$

and

where

$$
B(k, \mu, a)=(\mu+k) Z_{2}(k, \mu, a)
$$

$$
Z_{2}(k, \mu, a)=\frac{-2 J_{l}(\mu a)+\pi a J_{l}(k a)\left(\mu J_{l}^{\prime}(\mu a) Y_{l}(k a)-k J_{l}(\mu a) Y_{l}^{\prime}(k a)\right)}{2 a J_{l}(k a)\left[\mu J_{l}(k a) J_{l}^{\prime}(\mu a)-k J_{l}^{\prime}(k a) J_{l}(\mu a)\right]}
$$

then

and

$$
g_{l}^{+,+}\left(r, r^{\prime}\right)=-\pi\left[J_{l}\left(k r^{\prime}\right) Y_{l}(k r)-\frac{2 Z_{2}}{\pi} J_{l}\left(k r^{\prime}\right) J_{l}(k r)\right]
$$

$$
g_{l}^{-,-}\left(r, r^{\prime}\right)=-\pi\left[J_{l}\left(\mu r^{\prime}\right) Y_{l}(\mu r)-\frac{2 Z_{1}}{\pi} J_{l}\left(\mu r^{\prime}\right) J_{l}(\mu r)\right]
$$

Mixing cases:

The case $r^{\prime}<a<r$ : $r^{\prime}$ inside the disk and $\mathrm{r}$ outside the disk Following (28) we consider the pole $\tau=\mu$ and we obtain

$$
\begin{gathered}
G_{l}^{-}\left(p, r^{\prime}\right)=J_{l}\left(\mu r^{\prime}\right)\left[\frac{2 C(k, \mu, a)}{p-k} f(p)+\frac{h(p)}{(\mu+k)(p-k)}\right], \\
\text { where } f(p)=\frac{(p-\mu)(\mu+k)}{p+k} \text { and } h(p)=\frac{(-p)^{l}(\mu+k)}{k^{l}(p+k)}
\end{gathered}
$$

The inverse Hankel's transform applied to the equation (53) gives

$$
g_{l}^{+,-}\left(r, r^{\prime}\right)=J_{l}\left(\mu r^{\prime}\right) W(k, \mu, a, r)
$$

where

$$
W(k, \mu, a, r)=\left[2 C(k, \mu, a) \int_{0}^{+\infty} \frac{p(p-\mu) J_{l}(p r) d p}{p^{2}-k^{2}}+\frac{2}{(-k)^{l}} \int_{0}^{+\infty} \frac{p^{l+1} J_{l}(p r) d p}{p^{2}-k^{2}}\right]
$$

then after residue integration, we obtain

$$
g_{l}^{+,-}\left(r, r^{\prime}\right)=\pi J_{l}\left(\mu r^{\prime}\right)\left[-Y_{l}(k r)+C(k, \mu, a) J_{l}(k r)\right]
$$


Using the continuity of Green's function at point $r=a$

$$
g_{l}^{+,-}(a, a)=g_{l}^{+,+}(a, a)
$$

we have

$$
J_{l}(\mu a)\left[-Y_{l}(k a)+C(k, \mu, a) J_{l}(k a)\right]=\left[-Y_{l}(k a)+\frac{2 Z_{2}}{\pi} J_{l}(k a)\right] J_{l}(k a)
$$

from which we extract the constant $\mathrm{C}$

$$
C(k, \mu, a)=\frac{Y_{l}(k a)}{J_{l}(k a)}-\frac{4}{\pi a\left[\mu J_{l}(k a) J_{l}^{\prime}(\mu a)-k J_{l}^{\prime}(k a) J_{l}(\mu a)\right]}
$$

Finally we obtain the Green's function for the case r inside the disk and r' outside the disk:

$$
\left.g_{l}^{+,-}\left(r, r^{\prime}\right)=-\pi\left[Y_{l}(k r) J_{l}\left(\mu r^{\prime}\right)-C(k, \mu, a)\right] J_{l}(k r) J_{l}\left(\mu r^{\prime}\right)\right]
$$

The case $r<a<r^{\prime}: \mathrm{r}$ inside the disk and r' outside the disk Following the formula (34) at the pole $\tau=-k$

$$
\begin{gathered}
G_{l}^{+}\left(p, r^{\prime}\right)=-J_{l}\left(k r^{\prime}\right)\left[\frac{2 D(k, \mu, a)}{(\mu+k)(p+\mu)} T(p)+\frac{n(p)}{(\mu+k)(p+\mu)}\right] \\
\text { where } T(p)=\frac{(k-p)(\mu+k)}{(p-\mu)} \text {, and } n(p)=\frac{(-p)^{l-1}(\mu+k)}{k^{l}(p-\mu)}
\end{gathered}
$$

The inverse Hankel's transform applied to the last equation gives

$$
\begin{gathered}
g_{l}^{-,+}\left(r, r^{\prime}\right)=J_{l}\left(k r^{\prime}\right)\left[2 D(k, \mu, a) \int_{0}^{+\infty} \frac{p(p-k) J_{l}(p r) d p}{p^{2}-\mu^{2}}+\frac{2}{(-k)^{l}} \int_{0}^{+\infty} \frac{p^{l+1} J_{l}(p r) d p}{p^{2}-\mu^{2}}\right] \\
=\pi J_{l}\left(k r^{\prime}\right)\left[-Y_{l}(\mu r)+D(k, \mu, a) J_{l}(\mu r)\right]
\end{gathered}
$$

Respecting the continuity of the Green's function at $r=a$ we obtain

$$
\begin{gathered}
g_{l}^{-,+}(a, a)=g_{l}^{-,-}(a, a) \quad \text { then } \\
J_{l}(k a)\left[Y_{l}(\mu a)-D(k, \mu, a) J_{l}(\mu a)\right]=J_{l}(\mu a) Y_{l}(\mu a)- \\
\frac{-2 J_{l}(k a)+\pi a J_{l}(\mu a)\left(k J_{l}^{\prime}(k a) Y_{l}(\mu a)-\mu J_{l}(k a) Y_{l}^{\prime}(\mu a)\right)}{\pi a\left[k J_{l}^{\prime}(k a) J_{l}(\mu a)-\mu J_{l}(k a) J_{l}^{\prime}(\mu a)\right]} J_{l}(\mu a) \\
D(k, \mu, a)=\frac{Y_{l}(\mu a)}{J_{l}(\mu a)}-\frac{Y_{l}(\mu a)}{J_{l}(k a)}+ \\
\frac{-2 J_{l}(k a)+\pi a J_{l}(\mu a)\left(k J_{l}^{\prime}(k a) Y_{l}(\mu a)-\mu J_{l}(k a) Y_{l}^{\prime}(\mu a)\right)}{\pi a J_{l}(k a)\left[k J_{l}^{\prime}(k a) J_{l}(\mu a)-\mu J_{l}(k a) J_{l}^{\prime}(\mu a)\right]} \\
D(k, \mu, a)=\frac{Y_{l}(\mu a)}{J_{l}(\mu a)}-\frac{4}{\pi a\left[k J_{l}^{\prime}(k a) J_{l}(\mu a)-\mu J_{l}(k a) J_{l}^{\prime}(\mu a)\right]}
\end{gathered}
$$

then

$$
g_{l}^{-,+}\left(r, r^{\prime}\right)=-\pi\left[J_{l}\left(k r^{\prime}\right) Y_{l}(\mu r)-D(k, \mu, a) J_{l}\left(k r^{\prime}\right) J_{l}(\mu r)\right]
$$




\section{TWO-DIMENSIONAL WELL POTENTIAL}

This problem consists to consider in the Schrodinger equation, the potential $V(r)$ takes a negative value $\left(-V_{0}\right)$ inside the disk of radius a and takes zero outside the disk. To this problem, corresponds the following integral equation

$$
g_{l}\left(r, r^{\prime}\right)=g_{0}\left(r, r^{\prime}\right)+V_{0} \int_{0}^{a} \zeta g_{0}(r, \zeta) g_{l}\left(\zeta, r^{\prime}\right) d \zeta .
$$

Define as before two auxilliary functions $g^{+}(r)$ and $g^{-}(r)$ as it follows

$$
\begin{gathered}
g^{+}(r)=\left\{\begin{array}{cc}
g(r) & r \leq a \\
0 & r>a
\end{array}\right. \\
g^{-}(r)=\left\{\begin{array}{cc}
0 & r \leq a \\
-g(r) & r>a
\end{array}\right.
\end{gathered}
$$

after what, the equation (71) becomes as

$$
g_{l}^{+}\left(r, r^{\prime}\right)-g_{l}^{-}\left(r, r^{\prime}\right)=g_{0}\left(r, r^{\prime}\right)+V_{0} \int_{0}^{\infty} \zeta g_{0}(r, \zeta) g_{l}^{+}\left(\zeta, r^{\prime}\right) d \zeta .
$$

Now define Hankel's transformation of a function $g_{l}$ as

$$
\hat{G}_{l}\left(p, r^{\prime}\right)=\int_{0}^{\infty} r J_{l}(p r) g_{l}\left(r, r^{\prime}\right) d r
$$

and apply Hankel's transformation to the equation (74)

$$
\begin{aligned}
& \int_{0}^{\infty} r J_{l}(p r) g_{l}^{+}\left(r, r^{\prime}\right) d r-\int_{0}^{\infty} r J_{l}(p r) g_{l}^{-}\left(r, r^{\prime}\right) d r \\
= & \int_{0}^{\infty} r J_{l}(p r) g_{0}\left(r, r^{\prime}\right) d r+\int_{0}^{\infty} r J_{l}(p r) d r \int_{0}^{\infty} \zeta g_{0}(r, \zeta) g_{l}^{+}\left(\zeta, r^{\prime}\right) d \zeta
\end{aligned}
$$

Using the same method as above, we obtain

$$
\begin{gathered}
\hat{G}_{l}^{+}\left(p, r^{\prime}\right)-\hat{G}_{l}^{-}\left(p, r^{\prime}\right)=\frac{2 J_{l}\left(p r^{\prime}\right)}{p^{2}-k^{2}}+\frac{2 V_{0}}{p^{2}-k^{2}} \hat{G}_{l}^{+}\left(p, r^{\prime}\right) \\
\hat{G}_{l}^{+}\left(p, r^{\prime}\right)\left[\frac{p^{2}-\mu_{1}^{2}}{p^{2}-k^{2}}\right]-\hat{G}_{l}^{-}\left(p, r^{\prime}\right)=\frac{2 J_{l}\left(p r^{\prime}\right)}{p^{2}-k^{2}} \equiv B(p), \mu_{1}^{2}=k^{2}+2 V_{0}
\end{gathered}
$$

the fractional decomposition gives

$$
\frac{p^{2}-\mu_{1}^{2}}{p^{2}-k^{2}}=\frac{F^{+}}{F^{-}} \text {where } F^{+}(p)=\frac{p-\mu_{1}}{p-k}, \quad \text { and } \quad F^{-}(p)=\frac{p+k}{p+\mu_{1}}
$$

Respecting Hilbert's theorem the functions $\hat{G}_{l}^{+}$and $\hat{G}_{l}^{-}$are determinated by: 
for $r^{\prime} \leq a$

$$
\begin{aligned}
\hat{G}_{l}^{+}\left(p, r^{\prime}\right)=\frac{F^{-}(p)}{2 \pi i} \int_{L} \frac{B(\tau) d \tau}{F^{+}(\tau)(\tau-p)}, \quad \text { where } L \text { is a closed contour } \\
=\frac{p+k}{2 \pi i\left(p+\mu_{1}\right)} \int_{L} \frac{2 J_{l}\left(\tau r^{\prime}\right) d \tau}{\left(\tau^{2}-k^{2}\right)\left(\frac{\tau-\mu_{1}}{\tau-k}\right)(\tau-p)} \\
=\frac{p+k}{2 \pi i\left(p+\mu_{1}\right)} \int_{L} \frac{2 J_{l}\left(\tau r^{\prime}\right) d \tau}{(\tau+k)\left(\tau-\mu_{1}\right)(\tau-p)}
\end{aligned}
$$

apply the residue theorem at points ( $\tau=\mu_{1}$ and $\tau=p$ inside the contour) we obtain

$$
\hat{G}_{l}^{+}\left(p, r^{\prime}\right)=\frac{2 \beta\left(\mu_{1}, k, a\right)(p-k) J_{l}\left(\mu_{1} r^{\prime}\right)}{\left(\mu_{1}+k\right)\left(p^{2}-\mu_{1}^{2}\right)}+\frac{2 J_{l}\left(p r^{\prime}\right)}{p^{2}-\mu_{1}^{2}}
$$

The inverse Hankel's transform applied to the last equation, for $r \leq r^{\prime} \leq a$, gives

$$
\begin{gathered}
g^{++}\left(r, r^{\prime}\right)=\frac{2 \beta\left(\mu_{1}, k, a\right) J_{l}\left(\mu_{1} r^{\prime}\right)}{\mu_{1}+k} \int_{0}^{+\infty} \frac{p(p-k) J_{l}(p r) d p}{\left(p^{2}-\mu_{1}^{2}\right)}+2 \int_{0}^{+\infty} \frac{p J_{l}\left(p r^{\prime}\right) J_{l}(p r) d p}{\left(p^{2}-\mu_{1}^{2}\right)} \\
=\frac{2 \beta(k, \mu, a) J_{l}\left(\mu_{1} r^{\prime}\right) J_{l}\left(\mu_{1} r\right)}{\left(\mu_{1}+k\right)}-\pi J_{l}\left(\mu_{1} r^{\prime}\right) Y_{l}\left(\mu_{1} r\right)
\end{gathered}
$$

the same way for $r^{\prime}>a$

$$
\begin{gathered}
\hat{G}_{l}^{-}\left(p, r^{\prime}\right)=\frac{F^{+}(p)}{2 \pi i} \int_{L} \frac{B(\tau) d \tau}{F^{+}(\tau)(\tau-p)}, \quad \text { where } L \text { is a closed contour } \\
=\frac{p-\mu_{1}}{2 \pi i(p-k)} \int_{L} \frac{2 J_{l}\left(\tau r^{\prime}\right) d \tau}{(\tau+k)\left(\tau-\mu_{1}\right)(\tau-p)}
\end{gathered}
$$

apply the residue theorem at points ( $\tau=-k$ and $\tau=p$ inside the contour) we obtain

$$
\hat{G}_{l}^{-}\left(p, r^{\prime}\right)=\frac{2 \alpha\left(\mu_{1}, k, a\right)\left(p-\mu_{1}\right) J_{l}\left(k r^{\prime}\right)}{\left(\mu_{1}+k\right)\left(p^{2}-k^{2}\right)}+\frac{2 J_{l}\left(p r^{\prime}\right)}{p^{2}-k^{2}}
$$

The inverse Hankel's transform applied to the last equation, for the case $r>r^{\prime}>a$, gives

$$
\begin{gathered}
g^{--}\left(r, r^{\prime}\right)=\frac{2 \alpha\left(\mu_{1}, k, a\right) J_{l}\left(k r^{\prime}\right)}{\mu_{1}+k} \int_{0}^{+\infty} \frac{p\left(p-\mu_{1}\right) J_{l}(p r) d p}{\left(p^{2}-k^{2}\right)}+2 \int_{0}^{+\infty} \frac{p J_{l}\left(p r^{\prime}\right) J_{l}(p r) d p}{\left(p^{2}-k^{2}\right)} \\
=\frac{2 \alpha\left(\mu_{1}, k, a\right) J_{l}\left(k r^{\prime}\right) J_{l}(k r)}{\left(\mu_{1}+k\right)}-\pi J_{l}\left(k r^{\prime}\right) Y_{l}(k r)
\end{gathered}
$$

following the symmetry of the Green's function, we take $\alpha, \beta$ as function of $a$, then we obtain a sysem of two equations at $r=r^{\prime}=a$ 


$$
\begin{array}{r}
g_{l}^{++}(a, a)=g_{l}^{--}(a, a) \\
\left(d g_{l}^{++}(r, a) / d r\right)_{r=a}=\left(d g_{l}^{--}(r, a) / d r\right)_{r=a}
\end{array}
$$

that transforms as two coupled equations allowing to compute thereafter the coefficients $\alpha$ and $\beta$

$$
\begin{array}{r}
-\pi\left(\mu_{1}+k\right) J_{l}(k a) Y_{l}(k a)+2 \alpha\left(k, \mu_{1}, a\right) J_{l}(k a) J_{l}(k a) \\
=2 \beta\left(k, \mu_{1}, a\right) J_{l}\left(\mu_{1} a\right) J_{l}\left(\mu_{1} a\right)-\pi\left(\mu_{1}+k\right) J_{l}\left(\mu_{1} a\right) Y_{l}\left(\mu_{1} a\right) \\
-\pi k\left(\mu_{1}+k\right) Y_{l}^{\prime}(k a) J_{l}(k a)+2 k \alpha\left(k, \mu_{1}, a\right) J_{l}^{\prime}(k a) J_{l}(k a) \\
=2 \mu_{1} \beta\left(k, \mu_{1}, a\right) J_{l}\left(\mu_{1} a\right) J_{l}^{\prime}\left(\mu_{1} a\right)-\mu_{1} \pi\left(\mu_{1}+k\right) Y_{l}^{\prime}\left(\mu_{1} a\right) J_{l}\left(\mu_{1} a\right)
\end{array}
$$

Solving the equations (93-94), we find

$$
\frac{\beta\left(k, \mu_{1}, a\right)}{\mu_{1}+k}=\frac{-2 J_{l}(k a)+\pi a J_{l}\left(\mu_{1} a\right)\left(k J_{l}^{\prime}(k a) Y_{l}\left(\mu_{1} a\right)-\mu_{1} J_{l}(k a) Y_{l}^{\prime}\left(\mu_{1} a\right)\right)}{2 \pi a J_{l}\left(\mu_{1} a\right)\left(\mu_{1} J_{l}(k a) J_{l}^{\prime}\left(\mu_{1} a\right)-k J_{l}^{\prime}(k a) J_{l}\left(\mu_{1} a\right)\right)}
$$

and

$$
\frac{\alpha\left(k, \mu_{1}, a\right)}{\mu_{1}+k}=\frac{-2 J_{l}\left(\mu_{1} a\right)+\pi a J_{l}(k a)\left(\mu_{1} J_{l}^{\prime}\left(\mu_{1} a\right) Y_{l}(k a)-k J_{l}\left(\mu_{1} a\right) Y_{l}^{\prime}(k a)\right)}{2 \pi a J_{l}(k a)\left(k J_{l}^{\prime}(k a) J_{l}\left(\mu_{1} a\right)-\mu_{1} J_{l}(k a) J_{l}^{\prime}\left(\mu_{1} a\right)\right)}
$$

by which we find the compact forms of $g^{++}\left(r, r^{\prime}\right)$ and $g^{--}\left(r, r^{\prime}\right)$ in the following expressions

$$
g^{++}\left(r, r^{\prime}\right)=-\pi\left[Y_{l}\left(\mu_{1} r\right) J_{l}\left(\mu_{1} r^{\prime}\right)-\frac{\beta\left(k, \mu_{1}, a\right)}{\mu_{1}+k} J_{l}\left(\mu_{1} r\right) J_{l}\left(\mu_{1} r^{\prime}\right)\right]
$$

and

$$
g^{--}\left(r, r^{\prime}\right)=-\pi\left[Y_{l}(k r) J_{l}\left(k r^{\prime}\right)-\frac{\alpha\left(k, \mu_{1}, a\right)}{\mu_{1}+k} J_{l}(k r) J_{l}\left(k r^{\prime}\right)\right]
$$

We mention at this step, that the spectrum of the hamiltonian operator, is given by the poles of $g^{++}\left(r, r^{\prime}\right)$. Then the spectrum is given by putting the denominator of (95) equal zero. It is clearly that we have a transcendal equation to solve and then a numerical method is required at this stage to find the spectrum of the Hamiltonian. The cases $r^{\prime}<a<r$ and $r<a<r^{\prime}$ are also straightforward

$$
g_{l}^{+-}\left(r, r^{\prime}\right)=-\pi Y_{l}(k r) J_{l}\left(\mu_{1} r^{\prime}\right)+\pi\left[Q_{1}\right] J_{l}(k r) J_{l}\left(\mu_{1} r^{\prime}\right)
$$

where

$$
Q_{1}=\frac{Y_{l}(k a)}{J_{l}(k a)}-\frac{4}{\pi a\left[\mu_{1} J_{l}(k a) J_{l}^{\prime}\left(\mu_{1} a\right)-k J_{l}^{\prime}(k a) J_{l}\left(\mu_{1} a\right)\right]}
$$

and

$$
g_{l}^{-,+}\left(r, r^{\prime}\right)=-\pi J_{l}\left(k r^{\prime}\right) Y_{l}\left(\mu_{1} r\right)+\pi\left[Q_{2}\right] J_{l}\left(k r^{\prime}\right) J_{l}\left(\mu_{1} r\right)
$$


where

$$
Q_{2}=\frac{Y_{l}\left(\mu_{1} a\right)}{J_{l}\left(\mu_{1} a\right)}-\frac{4}{\pi a\left[k J_{l}^{\prime}(k a) J_{l}\left(\mu_{1} a\right)-\mu_{1} J_{l}(k a) J_{l}^{\prime}\left(\mu_{1} a\right)\right]}
$$

4.1. Infinite well problem. The following results are very required by physists (quantum mechanics) and mechanists. The two-dimensional problems serve as model to approach some realistic situation in differents problems encountred in physics and mechanics. For instance, in quantum mechanics, the energy spectra is the standard quantity to explore as it is very usefull to get all physical properties of the system. Usually, the physists solve the differential equation, as eigenvalues problem. Here we show the power tool of the integral equation in the demand of the eigen values of the Hamiltonian operator. Let the following integral equation

$$
\begin{gathered}
G_{l}\left(r, r^{\prime}\right)=G_{0}\left(r, r^{\prime}\right)+\int_{0}^{+\infty} \zeta G_{0}(r, \zeta) V(\zeta) G_{l}\left(\zeta, r^{\prime}\right) d \zeta \\
G_{l}\left(r, r^{\prime}\right)=G_{0}\left(r, r^{\prime}\right)+V_{0} \int_{a}^{+\infty} \zeta G_{0}(r, \zeta) \delta(\zeta-a) G_{l}\left(\zeta, r^{\prime}\right) d \zeta \\
G_{l}\left(r, r^{\prime}\right)=G_{0}\left(r, r^{\prime}\right)+a V_{0} G_{0}(r, a) G_{l}\left(a, r^{\prime}\right)
\end{gathered}
$$

for $r=a$, we have

$$
\begin{gathered}
G_{l}\left(a, r^{\prime}\right)=G_{0}\left(a, r^{\prime}\right)+a V_{0} G_{0}(a, a) G_{l}\left(a, r^{\prime}\right) \\
G_{l}\left(a, r^{\prime}\right)=\frac{G_{0}\left(a, r^{\prime}\right)}{1-a V_{0} G_{0}(a, a)}
\end{gathered}
$$

by substituting the last equation in (105) we get

$$
G_{l}\left(r, r^{\prime}\right)=G_{0}\left(r, r^{\prime}\right)+\frac{a V_{0} G_{0}(r, a) G_{0}\left(a, r^{\prime}\right)}{1-a V_{0} G_{0}(a, a)}
$$

when $V_{0}$ goes to infinity

$$
G_{l}\left(r, r^{\prime}\right)=G_{0}\left(r, r^{\prime}\right)-\frac{G_{0}(r, a) G_{0}\left(a, r^{\prime}\right)}{G_{0}(a, a)}
$$

This result is well known in differents papers [16]

The case $r<r^{\prime}<a$ :

$$
G_{0}\left(r, r^{\prime}\right)=J_{l}(k r) H_{l}^{(1)}\left(k r^{\prime}\right)
$$

therefore

$$
\begin{gathered}
G_{l}\left(r, r^{\prime}\right)=J_{l}(k r) H_{l}^{(1)}\left(k r^{\prime}\right)-\frac{J_{l}(k r) J_{l}\left(k r^{\prime}\right) H_{l}^{(1)}(k a)}{J_{l}(k a)} \\
G_{l}\left(r, r^{\prime}\right)=J_{l}(k r) H_{l}^{(1)}\left(k r^{\prime}\right)-J_{l}(k r) J_{l}\left(k r^{\prime}\right)\left[1+i \frac{Y_{l}(k a)}{J_{l}(k a)}\right] \\
G_{l}\left(r, r^{\prime}\right)=i J_{l}(k r)\left[Y_{l}\left(k r^{\prime}\right)-\frac{Y_{l}(k a)}{J_{l}(k a)} J_{l}\left(k r^{\prime}\right)\right]
\end{gathered}
$$


the case $r^{\prime}<r<a$ :

$$
G_{l}\left(r, r^{\prime}\right)=J_{l}\left(k r^{\prime}\right) H_{l}^{(1)}(k r)-J_{l}(k r) J_{l}\left(k r^{\prime}\right)\left[1+i \frac{Y_{l}(k a)}{J_{l}(k a)}\right]
$$

then

$$
G_{l}\left(r, r^{\prime}\right)=i J_{l}\left(k r^{\prime}\right)\left[Y_{l}(k r)-\frac{Y_{l}(k a)}{J_{l}(k a)} J_{l}(k r)\right]
$$

We retrieve then the results (113) and (115) by the use of the integral equation theory. These results agree with the well known ones in the litterature [17]. In this case, the spectrum is given by the poles of (115) that is to say by the root of the transcendal equation $J_{l}(k a)=0$, a result well known in quantum mechanics.

\section{CONCLUSION}

In this work we have calculated the Green's function relative to the timeindependent Schroedinger equation. The potential part in the Hamiltonian is a piecewise continuous operator obeying to a circular symmetry. We have used transformed the Schroedinger equation to an integral equation. As the problem have a circular symmetry, the polar coordinates are the adequate one tu use for this kind of problems. To solve the resulted integral equation, we have used successfully the Bessel's transform. We have, with the help of this technique, to retrieve the Green's function of two problems: the irst one is related to a potential equal zero operator on a disk of radius "a" and a positive constant $V_{0}$ outside this disk. The second is related to a potential equal a negative constant $-V_{0}$ on the disk of radius "a" and equal zero outside this disk. For each problem, we have calculated explicitely the Green's function in different regions of the plan. We have respected the boundary conditions of the problems. The particular case of an infinite well has been derived as a limit case of the second problem. The discrete spectra of the Hamiltonian operator have been also derived.

\section{REFERENCES}

[1] Melnikov Y. A., On a Test and planar movment Eng. Anal. Bound. Elem. 25 669-676 (2001).

[2] Ben Ali B and Meftah M.T., Explicit quantum Green's functions on a piecewise continuous symmetrical spherical potential Rep. Math. Phys. 74 73-87 (2014).

[3] Kukla S., Siedlecka U. and Zamorska I., On a Test and planar movments, Sci. Res. Inst. Math. Computer Sci. 11 (1), 53 (2012).

[4] Kukla S., On a Test and planar movment alone $x$, Sci. Res. Inst. Math. Computer Sci. 9 (1), 77 (2010).

[5] Adhikari S. K.: Am. J. Phys. 54362367 (1986).

[6] Nemenman I. M.,Silbergleit A. S.: J. Appl. Phys. 86, No. 1614624 (1999)

[7] Yoshio Nosaka J.: Phys. Chem. 9550545058 (1991).

[8] Layeghnejad R., Zare M. and Moazzemi R.: Phys. Rev. D 84125026 (2011).

[9] Tago Y., Narasimha K.: Phys. Lett. 45A, No. 13738 (1973).

[10] Berezin F. A. and Shubin M. A. The Schroedinger Equation. Kluwer Academic Publishers, 1983.

[11] Feynman R. P. and Hibbs A. R.: Quantum Mechanics and Path Integrals (1965) (New York: McGraw Hill). 
[12] Dautray R., Louis Lions J., Michel Artola, Philippe Benilan and Michel Bernadou, Michel Cessinat, Jean-Claude Nedecel, Planchard J., Analyse mathmatique et calcul numrique, Masson (1984).

[13] Bhagwat K.V. and Lawande S.V., Perturbation in path integrals; Physics Letters A, 141, 321 (1989).

[14] Gradshteyn I. S. and Ryzhik I. M. Table of Integrals, Series, and Products 5th edition ed A Jeffrey (Boston Academic) (1994)

[15] Smirnov A., A course of high Mathematics, Volume IV, translated by Brown D. E., Pergamon Press, 1964.

[16] Groshe C.: Phys. Rep., No. 13738 (1973).

[17] Selcuck A., mathematical Methods in science and engineering, (2006) Edition A John Wiley and sons, inc, Publication. 